\title{
ON GAUSS-KRONROD QUADRATURE FORMULAE OF CHEBYSHEV TYPE
}

\author{
SOTIRIOS E. NOTARIS
}

\begin{abstract}
We prove that there is no positive measure $d \sigma$ on $(a, b)$ such that the corresponding Gauss-Kronrod quadrature formula is also a Chebyshev quadrature formula. The same is true if we consider measures of the form $d \sigma(t)=\omega(t) d t$, where $\omega(t)$ is even, on a symmetric interval $(-a, a)$, and the Gauss-Kronrod formula is required to have equal weights only for $n$ even. We also show that the only positive and even measure $d \sigma(t)=d \sigma(-t)$ on $(-1,1)$ for which the Gauss-Kronrod formula has all weights equal if $n=1$, or has the form $\int_{-1}^{1} f(t) d \sigma(t)=w \sum_{\nu=1}^{n} f\left(\tau_{\nu}\right)+w_{1} f(1)+w \sum_{\mu=2}^{n} f\left(\tau_{\mu}^{*}\right)+$ $w_{1} f(-1)+R_{n}^{K}(f)$ for all $n \geq 2$, is the Chebyshev measure of the first kind $d \sigma_{C}(t)=\left(1-t^{2}\right)^{-1 / 2} d t$.
\end{abstract}

\section{INTRODUCTION}

Let $d \sigma$ be a positive measure on the interval $(a, b)$, whose moments all exist,

$$
\mu_{i}=\int_{a}^{b} t^{i} d \sigma(t)<\infty, \quad i=0,1,2, \ldots
$$

The Gauss-Kronrod quadrature formula for $d \sigma$ has the form

$$
\int_{a}^{b} f(t) d \sigma(t)=\sum_{\nu=1}^{n} \sigma_{\nu} f\left(\tau_{\nu}\right)+\sum_{\mu=1}^{n+1} \sigma_{\mu}^{*} f\left(\tau_{\mu}^{*}\right)+R_{n}^{K}(f),
$$

where $\tau_{\nu}=\tau_{\nu}^{(n)}$ are the zeros of the $n$ th-degree (monic) orthogonal polynomial $\pi_{n}(\cdot)=\pi_{n}(\cdot ; d \sigma)$, and the $\tau_{\mu}^{*}=\tau_{\mu}^{*(n)}, \sigma_{\nu}=\sigma_{\nu}^{(n)}$, and $\sigma_{\mu}^{*}=\sigma_{\mu}^{*(n)}$ are determined such that (1.2) has maximum degree of exactness (at least) $3 n+1$, i.e., $R_{n}^{K}(f)=0$ for all $f \in \mathbb{P}_{3 n+1}$. Then the $\tau_{\mu}^{*}$ must be the zeros of a (monic) polynomial $\pi_{n+1}^{*}(\cdot)=\pi_{n+1}^{*}(\cdot ; d \sigma)$, called the Stieltjes polynomial, which satisfies the orthogonality condition

$$
\int_{a}^{b} \pi_{n}(t) \pi_{n+1}^{*}(t) t^{i} d \sigma(t)=0, \quad i=0,1, \ldots, n,
$$

that is, $\pi_{n+1}^{*}$ is orthogonal to all polynomials of lower degree with respect to the oscillatory measure $d \sigma^{*}(t)=\pi_{n}(t) d \sigma(t)$ on $(a, b)$. It can be shown that

Received March 2, 1990; revised January 31, 1991.

1991 Mathematics Subject Classification. Primary 65D32; Secondary 33C45.

Key words and phrases. Gauss-Kronrod quadrature formulae, Chebyshev quadrature. 
$\pi_{n+1}^{*}$ is uniquely defined by $(1.3)$ (see $\left.[1, \S 4]\right)$. However, since $d \sigma^{*}$ changes sign on $(a, b)$, the reality of the $\tau_{\mu}^{*}$ cannot in general be assumed.

A quadrature rule

$$
\int_{a}^{b} f(t) d \sigma(t)=\sum_{i=1}^{n} w_{i} f\left(t_{i}\right)+R_{n}^{C}(f)
$$

with equal weights

$$
w_{1}^{(n)}=w_{2}^{(n)}=\cdots=w_{n}^{(n)}
$$

is called a Chebyshev quadrature rule, if the nodes $t_{k}=t_{k}^{(n)}$ are real and if (1.4) has degree of exactness (at least) $n$. By setting $f(t)=1$ in (1.4), we find, in view of $(1.5)$,

$$
w_{i}=\frac{\mu_{0}}{n}, \quad i=1,2, \ldots, n .
$$

It is well known that the only equally weighted (for all $n$ ) Gauss formula is the one relative to the Chebyshev measure of the first kind, $d \sigma_{C}(t)=$ $\left(1-t^{2}\right)^{-1 / 2} d t$ on $(-1,1)$ (see, e.g., $[2, \S 4]$ ). If the Gauss formula has the equicoefficient property only for $n$ even, then among the positive measures of the form $d \sigma(t)=\omega(t) d t$, where $\omega(t)$ is even, with symmetric support, the only one admitting such a formula is, up to a linear transformation,

$$
d \sigma_{\xi}(t)=\left\{\begin{array}{l}
|t|\left(t^{2}-\xi^{2}\right)^{-1 / 2}\left(1-t^{2}\right)^{-1 / 2} d t, \quad t \in[-1,-\xi] \cup[\xi, 1], \\
0 \quad \text { elsewhere }
\end{array}\right.
$$

$($ see $[3, \S 6])$.

The only Gauss-Kronrod formula known, which is almost of Chebyshev type, is the one relative to the Chebyshev measure of the first kind,

$$
\begin{aligned}
\int_{-1}^{1} f(t)\left(1-t^{2}\right)^{-1 / 2} d t= & \frac{\pi}{3} \sum_{i=1}^{3} f\left(\cos \frac{2 i-1}{6} \pi\right)+R_{1}^{K C}(f) \\
\int_{-1}^{1} f(t)\left(1-t^{2}\right)^{-1 / 2} d t= & \frac{\pi}{2 n}\left[\frac{1}{2} f(-1)+\sum_{i=1}^{2 n-1} f\left(\cos \frac{i \pi}{2 n}\right)+\frac{1}{2} f(1)\right] \\
& +R_{n}^{K C}(f), \quad n \geq 2
\end{aligned}
$$

(see, e.g., [6, equation (43)]). Incidentally, the second formula in (1.8) is the same as the Gauss-Lobatto formula for $d \sigma_{C}(t)=\left(1-t^{2}\right)^{-1 / 2} d t$ with $2 n+1$ points, hence it has elevated degree of exactness $4 n-1$.

Equally-weighted quadrature rules are useful in practice because they minimize the effect of random errors in the values of $f\left(t_{i}\right)$ in (1.4). Therefore, it is interesting to examine if there are positive measures admitting GaussKronrod formulae of Chebyshev type. In the next section we show that such measures do not exist. This is also the case if we consider measures of the form $d \sigma(t)=\omega(t) d t$, where $\omega(t)$ is even, with symmetric support, and the GaussKronrod formula is required to have equal weights only for $n$ even. Naturally, one then wonders if there are at least other Gauss-Kronrod formulae of the form (1.8). In $\S 3$, we prove that the only positive and even measure $d \sigma(t)=d \sigma(-t)$ on $(-1,1)$, which gives rise to a Gauss-Kronrod formula of the type (1.8), is $d \sigma_{C}(t)=\left(1-t^{2}\right)^{-1 / 2} d t$. In both sections we follow the technique used by 
Geronimus [4, 5] to show that the only Gauss formula of Chebyshev type is the one relative to the Chebyshev measure of the first kind.

\section{Nonexistence of GaUSS-KRONROD QuAdRATURE fORMUlaE OF CHEBYSHEV TYPE}

It is known that for any positive measure $d \sigma$ on $(a, b)$ the respective (monic) orthogonal polynomials $\left\{\pi_{i}(\cdot ; d \sigma)\right\}$ satisfy a three-term recurrence relation of the form

$$
\begin{aligned}
& \pi_{-1}(t)=0, \quad \pi_{0}(t)=1, \\
& \pi_{i+1}(t)=\left(t-\alpha_{i}\right) \pi_{i}(t)-\beta_{i} \pi_{i-1}(t), \quad i=0,1,2, \ldots,
\end{aligned}
$$

where the recursion coefficients $\alpha_{i}=\alpha_{i}(d \sigma)$ and $\beta_{i}=\beta_{i}(d \sigma)$ are given by the formulae

$$
\begin{aligned}
& \alpha_{i}=\frac{\int_{a}^{b} t\left[\pi_{i}(t)\right]^{2} d \sigma(t)}{\int_{a}^{b}\left[\pi_{i}(t)\right]^{2} d \sigma(t)}, \quad i=0,1,2, \ldots, \\
& \beta_{0}=\int_{a}^{b} d \sigma(t), \quad \beta_{i}=\frac{\int_{a}^{b}\left[\pi_{i}(t)\right]^{2} d \sigma(t)}{\int_{a}^{b}\left[\pi_{i-1}(t)\right]^{2} d \sigma(t)}, \quad i=1,2, \ldots,
\end{aligned}
$$

hence $\beta_{i}>0, \quad i=0,1,2, \ldots$. Using (2.1) and induction, we can show that

$$
\begin{aligned}
\pi_{n}(t)= & t^{n}-\left(\sum_{i=0}^{n-1} \alpha_{i}\right) t^{n-1} \\
& +\left(\sum_{\substack{i, j=0 \\
i<j}}^{n-1} \alpha_{i} \alpha_{j}-\sum_{i=1}^{n-1} \beta_{i}\right) t^{n-2}+\cdots, \quad n \geq 1 .
\end{aligned}
$$

A similar formula can be obtained for the corresponding Stieltjes polynomial.

Lemma 2.1. The Stieltjes polynomial $\pi_{n+1}^{*}(\cdot ; d \sigma)$ has the form

$$
\begin{aligned}
\pi_{n+1}^{*}(t)= & t^{n+1}-\left(\sum_{i=0}^{n} \alpha_{i}\right) t^{n} \\
& +\left(\sum_{\substack{i, j=0 \\
i<j}}^{n} \alpha_{i} \alpha_{j}-\sum_{i=1}^{n+1} \beta_{i}\right) t^{n-1}+\cdots, \quad n \geq 1 .
\end{aligned}
$$

Proof. Expanding $\pi_{n+1}^{*}$ in terms of $\pi_{i}$, we have

$$
\pi_{n+1}^{*}(t)=\pi_{n+1}(t)+c_{0} \pi_{n}(t)+\cdots+c_{n-1} \pi_{1}(t)+c_{n} \pi_{0}(t)
$$

and then substituting into (1.3) with $i=0,1$ yields, by means of (2.2) and orthogonality,

$$
c_{0}=0, \quad c_{1}=-\beta_{n+1} .
$$

These, together with (2.3) and (2.5), imply (2.4).

We can now prove our main result. 
Theorem 2.2. There is no positive measure $d \sigma$ on $(a, b)$ relative to which (1.2) is also a Chebyshev quadrature formula for each $n=1,2, \ldots$.

Proof. Assume that there exists a positive measure $d \sigma$ on $(a, b)$ for which (1.2) is a Chebyshev quadrature formula, that is, has the form

$$
\int_{a}^{b} f(t) d \sigma(t)=\frac{\mu_{0}}{2 n+1}\left[\sum_{\nu=1}^{n} f\left(\tau_{\nu}\right)+\sum_{\mu=1}^{n+1} f\left(\tau_{\mu}^{*}\right)\right]+R_{n}^{K}(f) .
$$

Since for each $n=1,2, \ldots$, (2.7) is exact for $f(t)=t$ and $f(t)=t^{2}$, we obtain

$$
\sum_{\nu=1}^{n} \tau_{\nu}+\sum_{\mu=1}^{n+1} \tau_{\mu}^{*}=(2 n+1) m_{1}, \quad \sum_{\nu=1}^{n} \tau_{\nu}^{2}+\sum_{\mu=1}^{n+1} \tau_{\mu}^{* 2}=(2 n+1) m_{2}^{2},
$$

where $m_{1}=\mu_{1} / \mu_{0}$ and $m_{2}^{2}=\mu_{2} / \mu_{0}$. Let

$$
p_{2 n+1}(t)=\pi_{n}(t) \pi_{n+1}^{*}(t)
$$

with $\pi_{n}(t)=\prod_{\nu=1}^{n}\left(t-\tau_{\nu}\right)$ and $\pi_{n+1}^{*}(t)=\prod_{\mu=1}^{n+1}\left(t-\tau_{\mu}^{*}\right)$. First, because of $(2.8)$ we must have

$$
p_{2 n+1}(t)=t^{2 n+1}-(2 n+1) m_{1} t^{2 n}+\frac{2 n+1}{2}\left[(2 n+1) m_{1}^{2}-m_{2}^{2}\right] t^{2 n-1}+\cdots
$$

Also, substituting $\pi_{n}(t)$ and $\pi_{n+1}^{*}(t)$ in (2.9) from (2.3) and (2.4), one finds, after a simple computation,

$$
\begin{aligned}
p_{2 n+1}(t)= & t^{2 n+1}-\left(2 \sum_{i=0}^{n-1} \alpha_{i}+\alpha_{n}\right) t^{2 n} \\
& +\left[\left(\sum_{i=0}^{n-1} \alpha_{i}\right)^{2}+2 \sum_{\substack{i, j=0 \\
i<j}}^{n} \alpha_{i} \alpha_{j}-\left(2 \sum_{i=1}^{n-1} \beta_{i}+\beta_{n}+\beta_{n+1}\right)\right] t^{2 n-1}+\cdots
\end{aligned}
$$

Equating the coefficients of $t^{2 n}$ and $t^{2 n-1}$ gives

$$
\begin{gathered}
2 \sum_{i=0}^{n-1} \alpha_{i}+\alpha_{n}=(2 n+1) m_{1}, \quad n \geq 1, \\
\left(\sum_{i=0}^{n-1} \alpha_{i}\right)^{2}+2 \sum_{\substack{i, j=0 \\
i<j}}^{n} \alpha_{i} \alpha_{j}-\left(2 \sum_{i=1}^{n-1} \beta_{i}+\beta_{n}+\beta_{n+1}\right) \\
=\frac{2 n+1}{2}\left[(2 n+1) m_{1}^{2}-m_{2}^{2}\right], \quad n \geq 1 .
\end{gathered}
$$

Now from (2.2) we find

$$
\alpha_{0}=m_{1}, \quad \beta_{1}=m_{2}^{2}-m_{1}^{2},
$$


which, inserted into (2.12), yields, for $n=1$,

$$
\alpha_{1}=m_{1}, \quad \beta_{2}=\frac{1}{2}\left(m_{2}^{2}-m_{1}^{2}\right),
$$

and for $n=2$,

$$
\alpha_{2}=m_{1}, \quad \beta_{3}=0 .
$$

This contradicts the fact that $\beta_{3}>0$.

The negative result of Theorem 2.2 leads us to explore the possibility of having Gauss-Kronrod formulae with equal weights only for $n$ even. We restrict our search among the positive measures of the type $d \sigma(t)=\omega(t) d t$, where $\omega(t)$ is even, with symmetric support $(-a, a)$. Thus, we want $(1.2)$ to have the form

$$
\int_{-a}^{a} f(t) \omega(t) d t=\frac{\mu_{0}}{2 n+1}\left[\sum_{\nu=1}^{n} f\left(\tau_{\nu}\right)+\sum_{\mu=1}^{n+1} f\left(\tau_{\mu}^{*}\right)\right]+R_{n}^{K}(f), \quad n=2 k
$$

Since $d \sigma$ is an even measure with symmetric support, using orthogonality and (1.3), one easily shows by uniqueness that $\pi_{n}$ and $\pi_{n+1}^{*}$ are always either even or odd depending on the parity of $n$, that is,

$$
\begin{aligned}
& \pi_{n}(-t)=(-1)^{n} \pi_{n}(t), \quad n=0,1,2, \ldots, \\
& \pi_{n+1}^{*}(-t)=(-1)^{n+1} \pi_{n+1}^{*}(t), \quad n=0,1,2, \ldots .
\end{aligned}
$$

Consequently, the $\tau_{\nu}$ and $\tau_{\mu}^{*}$ in (2.16) are symmetric with respect to the origin. Setting $f(t)=g\left(t^{2}\right), g \in \mathbb{P}_{[(3 n+1) / 2]}$, where $[\cdot]$ denotes the integer part of a real number, it follows by symmetry that

$$
\int_{0}^{a} g\left(t^{2}\right) \omega(t) d t=\frac{\mu_{0}}{4 k+1}\left[\sum_{\nu=1}^{k} g\left(\tau_{\nu}^{2}\right)+\sum_{\mu=1}^{k} g\left(\tau_{\mu}^{* 2}\right)+\frac{1}{2} g(0)\right]_{\text {for all } g \in \mathbb{P}_{3 k} .}
$$

Letting $t=x^{1 / 2}$, so that $d t=\frac{1}{2} x^{-1 / 2} d x$, we get

$$
\begin{array}{rl}
\int_{0}^{a^{2}} & g(x) \omega\left(x^{1 / 2}\right) x^{-1 / 2} d x \\
& =\frac{2 \mu_{0}}{4 k+1}\left[\sum_{\nu=1}^{k} g\left(\tau_{\nu}^{2}\right)+\sum_{\mu=1}^{k} g\left(\tau_{\mu}^{* 2}\right)+\frac{1}{2} g(0)\right] \quad \text { for all } g \in \mathbb{P}_{3 k} .
\end{array}
$$

If $\bar{\omega}(x)=\omega\left(x^{1 / 2}\right) x^{-1 / 2}$ and $\bar{a}=a^{2}$, then $\bar{\mu}_{0}=\mu_{0}, \bar{\tau}_{\nu}=\tau_{\nu}^{2}$, and $\bar{\tau}_{\mu}^{*}=\tau_{\mu}^{* 2}$, where for the rest of this section all the quantities carrying a bar refer to the measure $d \bar{\sigma}(x)=\bar{\omega}(x) d x$ on $(0, \bar{a})$. Replacing $x$ by $t$ in (2.19), we obtain

$$
\int_{0}^{\bar{a}} g(t) \bar{\omega}(t) d t=\frac{\bar{\mu}_{0}}{2 k+1 / 2}\left[\sum_{\nu=1}^{k} g\left(\bar{\tau}_{\nu}\right)+\sum_{\mu=1}^{k} g\left(\bar{\tau}_{\mu}^{*}\right)+\frac{1}{2} g(0)\right]
$$

for all $g \in \mathbb{P}_{3 k}$.

Therefore, it comes down to examining if there exist Gauss-Kronrod formulae of the type (2.20), that is, one of the zeros of $\bar{\pi}_{k+1}^{*}$ is 0 , and all the weights are equal except the one corresponding to the node at 0 . 
Our findings are given in the following:

Theorem 2.3. There is no positive measure of the form $d \sigma(t)=\omega(t) d t$, where $\omega(t)$ is even, with symmetric support $(-a, a)$, for which (1.2) has equal weights for all $n$ even.

Proof. To prove the theorem, it suffices to show that there is no Gauss-Kronrod formula of the type (2.20). If we assume that such a formula exists, by much the same way as in the proof of Theorem 2.2 , we find that $\bar{p}_{2 k+1}$ (cf. (2.9)) must have the form

$$
\begin{aligned}
\bar{p}_{2 k+1}(t)= & t^{2 k+1}-\left(2 k+\frac{1}{2}\right) \bar{m}_{1} t^{2 k} \\
& +\frac{2 k+1 / 2}{2}\left[\left(2 k+\frac{1}{2}\right) \bar{m}_{1}^{2}-\bar{m}_{2}^{2}\right] t^{2 k-1}+\cdots,
\end{aligned}
$$

where $\bar{m}_{1}=\bar{\mu}_{1} / \bar{\mu}_{0}$ and $\bar{m}_{2}^{2}=\bar{\mu}_{2} / \bar{\mu}_{0}$. Then from (2.21) and (2.11), with $\bar{p}_{2 k+1}$ in place of $p_{2 n+1}$, we obtain the equations

$$
\begin{aligned}
& 2 \sum_{i=0}^{k-1} \bar{\alpha}_{i}+\bar{\alpha}_{k}=\left(2 k+\frac{1}{2}\right) \bar{m}_{1}, \quad k \geq 1, \\
& \left(\sum_{i=0}^{k-1} \bar{\alpha}_{i}\right)^{2}+2 \sum_{\substack{i, j=0 \\
i<j}}^{k} \bar{\alpha}_{i} \bar{\alpha}_{j}-\left(2 \sum_{i=1}^{k-1} \bar{\beta}_{i}+\bar{\beta}_{k}+\bar{\beta}_{k+1}\right) \\
& =\frac{2 k+1 / 2}{2}\left[\left(2 k+\frac{1}{2}\right) \bar{m}_{1}^{2}-\bar{m}_{2}^{2}\right], \quad k \geq 1,
\end{aligned}
$$

where $\bar{\alpha}_{i}=\alpha_{i}(d \bar{\sigma})$ and $\bar{\beta}_{i}=\beta_{i}(d \bar{\sigma})$. From (2.13) we have

$$
\bar{\alpha}_{0}=\bar{m}_{1}, \quad \bar{\beta}_{1}=\bar{m}_{2}^{2}-\bar{m}_{1}^{2} \text {. }
$$

Then (2.22) gives, for $k=1$,

$$
\bar{\alpha}_{1}=\frac{1}{2} \bar{m}_{1}, \quad \bar{\beta}_{2}=\frac{1}{4}\left(\bar{m}_{2}^{2}-\frac{1}{2} \bar{m}_{1}^{2}\right),
$$

and for $k=2$,

$$
\bar{\alpha}_{2}=\frac{3}{2} \bar{m}_{1}, \quad \bar{\beta}_{3}=-\frac{1}{4} \bar{m}_{1}^{2},
$$

which contradicts the fact that $\bar{\beta}_{3}>0$.

\section{GAUSS-KRONROD QUADRATURE FORMULAE ALMOST OF CHEBYSHEV TYPE}

Throughout this section $d \sigma$ is a positive and even measure $d \sigma(t)=d \sigma(-t)$ on $(-1,1)$. Then some of the quantities and formulae of the previous sections take a special form. First, it is clear from (1.1) that

$$
\mu_{i}=0 \text { for all } i \text { odd. }
$$

Also, $\pi_{i}$ is either even or odd depending on the parity of $i$ (cf. (2.17)). Then it follows from (2.2) that

$$
\alpha_{i}=0, \quad i=0,1,2, \ldots .
$$


Moreover, (2.4) with $n=1$ implies

$$
\pi_{2}^{*}(t)=t^{2}-\left(\beta_{1}+\beta_{2}\right)
$$

hence (cf. (2.9) with $n=1$ )

$$
p_{3}(t)=\pi_{1}(t) \pi_{2}^{*}(t)=t^{3}-\left(\beta_{1}+\beta_{2}\right) t=\pi_{3}(t),
$$

where for the last equality in (3.4) we used (2.3) and (2.17). Therefore, for $n=1$, the Gauss-Kronrod formula is the 3-point Gauss formula. (The same is true if the support of $d \sigma$ is any interval symmetric with respect to the origin.) We want to determine if there are any other positive and even measures $d \sigma$ on $(-1,1)$, besides the Chebyshev measure of the first kind, for which the Gauss-Kronrod formula has the form

$$
\begin{aligned}
\int_{-1}^{1} f(t) d \sigma(t)= & \frac{\mu_{0}}{3}\left[f\left(\tau_{1}\right)+f\left(\tau_{1}^{*}\right)+f\left(\tau_{2}^{*}\right)\right]+R_{1}^{K}(f), \\
\int_{-1}^{1} f(t) d \sigma(t)= & w \sum_{\nu=1}^{n} f\left(\tau_{\nu}\right)+w_{1} f(-1) \\
& +w \sum_{\mu=2}^{n} f\left(\tau_{\mu}^{*}\right)+w_{1} f(1)+R_{n}^{K}(f), \quad n \geq 2,
\end{aligned}
$$

that is, for all $n \geq 2$ two of the zeros of $\pi_{n+1}^{*}$ are \pm 1 , and all the weights are equal except those corresponding to the nodes at \pm 1 .

The existence of quadrature formulae of this kind is described in the following:

Theorem 3.1. The only positive and even measure $d \sigma$ on $(-1,1)$ for which the Gauss-Kronrod quadrature formula has the form (3.5) is the Chebyshev measure of the first kind $d \sigma_{C}(t)=\left(1-t^{2}\right)^{-1 / 2} d t$.

Proof. We proceed along the lines of the proof of Theorem 2.2. Assume that for the positive and even measure $d \sigma$ on $(-1,1)$ the Gauss-Kronrod formula is of the type (3.5). First, (3.1) implies that $m_{1}=\mu_{1} / \mu_{0}=0$. Then from (2.13) we find

$$
\beta_{1}=m_{2}^{2},
$$

where $m_{2}^{2}=\mu_{2} / \mu_{0}$, and for $n=1$, as in the proof of Theorem 2.2 (cf. $(2.14))$, we get

$$
\beta_{2}=\frac{1}{2} m_{2}^{2}
$$

If $n \geq 2$, since $d \sigma$ is an even measure with symmetric support, the $\tau_{\nu}$ and $\tau_{\mu}^{*}$ in the second formula in (3.5) are symmetric with respect to the origin. Also, this formula is exact for $f(t)=1$ and $f(t)=t^{2}$; hence we obtain, after setting $w_{1}=c w$,

$$
\begin{aligned}
& (2 n+2 c-1) w=\mu_{0}, \\
& w\left(\sum_{\nu=1}^{n} \tau_{\nu}^{2}+\sum_{\mu=2}^{n} \tau_{\mu}^{* 2}+2 c\right)=\mu_{2},
\end{aligned}
$$


from which it follows that

$$
\sum_{\nu=1}^{n} \tau_{\nu}^{2}+\sum_{\mu=2}^{n} \tau_{\mu}^{* 2}+2=(2 n+2 c-1) m_{2}^{2}+2(1-c) .
$$

Because of this, $p_{2 n+1}$ (cf. (2.9)) must have the form

$$
p_{2 n+1}(t)=t^{2 n+1}-\left[\left(n+c-\frac{1}{2}\right) m_{2}^{2}+1-c\right] t^{2 n-1}+\cdots .
$$

Moreover, from (2.11) and (3.2) we have

$$
p_{2 n+1}(t)=t^{2 n+1}-\left(2 \sum_{i=1}^{n-1} \beta_{i}+\beta_{n}+\beta_{n+1}\right) t^{2 n-1}+\cdots .
$$

By equating the coefficients of $t^{2 n-1}$, we derive the equation

$$
2 \sum_{i=1}^{n-1} \beta_{i}+\beta_{n}+\beta_{n+1}=\left(n+c-\frac{1}{2}\right) m_{2}^{2}+1-c, \quad n \geq 2 .
$$

Applying (3.12) for two successive values of $n$, and then subtracting the two equations, we get

$$
\beta_{n}+\beta_{n+2}=m_{2}^{2}, \quad n \geq 2,
$$

which, by means of $(3.7)$, gives

$$
\beta_{2 j}=\frac{1}{2} m_{2}^{2}, \quad j=1,2, \ldots .
$$

Now using (1.3) with $i=2,3,(2.1)$, and orthogonality, we find, after a lengthy but straightforward computation, that $c_{2}$ and $c_{3}$ in $(2.5)$, when $d \sigma$ is an even measure, are given by

$$
c_{2}=0, \quad c_{3}=\left(\beta_{n-1}-\beta_{n+2}\right) \beta_{n+1} .
$$

Then from $(2.5),(2.6),(3.15)$, and (2.1) we get analytic expressions for $\pi_{3}^{*}$ and $\pi_{4}^{*}$,

$$
\begin{aligned}
& \pi_{3}^{*}(t)=t^{3}-\left(\beta_{1}+\beta_{2}+\beta_{3}\right) t \\
& \pi_{4}^{*}(t)=t^{4}-\left(\beta_{1}+\beta_{2}+\beta_{3}+\beta_{4}\right) t^{2}+\beta_{1} \beta_{3}+\beta_{1} \beta_{4}+\beta_{2} \beta_{4}-\beta_{4} \beta_{5} .
\end{aligned}
$$

Since $\pi_{3}^{*}( \pm 1)=0$ and $\pi_{4}^{*}( \pm 1)=0$, we obtain the equations

$$
\begin{aligned}
& 1-\left(\beta_{1}+\beta_{2}+\beta_{3}\right)=0, \\
& 1-\left(\beta_{1}+\beta_{2}+\beta_{3}+\beta_{4}\right)+\beta_{1} \beta_{3}+\beta_{1} \beta_{4}+\beta_{2} \beta_{4}-\beta_{4} \beta_{5}=0 .
\end{aligned}
$$

These, together with

$$
\beta_{1}=2 \beta_{2}=2 \beta_{4}
$$

(cf. (3.6) and (3.14)), yield

$$
\beta_{4}\left(\beta_{3}-\beta_{5}\right)=0,
$$

which, on account of $\beta_{4}>0$, gives

$$
\beta_{3}=\beta_{5} \text {. }
$$

Then (3.13) implies

$$
\beta_{2 j+1}=\frac{1}{2} m_{2}^{2}, \quad j=1,2, \ldots,
$$


so finally

$$
\beta_{1}=m_{2}^{2}, \quad \beta_{i}=\frac{1}{2} m_{2}^{2}, \quad i=2,3, \ldots .
$$

Substituting $\beta_{1}, \beta_{2}, \beta_{3}$ from (3.22) into the first equation in (3.17), we obtain

$$
m_{2}^{2}=\frac{1}{2} \text {. }
$$

Therefore,

$$
\beta_{1}=\frac{1}{2}, \quad \beta_{i}=\frac{1}{4}, \quad i=2,3, \ldots,
$$

and $d \sigma$ is the Chebyshev measure of the first kind.

\section{ACKNOWLEDGMENT}

The author is indebted to Professor W. Gautschi for helpful suggestions and comments during the preparation of this paper.

\section{BIBLIOGRAPHY}

1. F. Caliò, W. Gautschi, and E. Marchetti, On computing Gauss-Kronrod quadrature formulae, Math. Comp. 47 (1986), 639-650.

2. W. Gautschi, Advances in Chebyshev quadrature, Numerical Analysis (G. A. Watson, ed.), Proc. Dundee Conf. on Numerical Analysis 1975, Lecture Notes in Math., vol. 506, SpringerVerlag, Berlin, 1976, pp. 100-121.

3. __ On some orthogonal polynomials of interest in theoretical chemistry, BIT 24 (1984), 473-483.

4. Ja. L. Geronimus, On Gauss' and Tchebycheff's quadrature formulas, Bull. Amer. Math. Soc. 50 (1944), 217-221.

5. _, On Gauss' and Tchebycheff's quadrature formulae, C.R. (Doklady) Akad. Sci. USSR (N. S.) 51 (1946), 655-658.

6. G. Monegato, Stieltjes polynomials and related quadrature rules, SIAM Rev. 24 (1982), $137-158$.

Department of Mathematical Sciences, Indiana University-Purdue University at INDIANAPOLIS, 1125 E. 38Th STREET, INDIANAPOLIS, INDIANA 46205

Current address: Department of Mathematics, University of Missouri-Columbia, Columbia, Missouri 65211

E-mail address: sotirios@notaris.cs.missouri.edu 